九州大学学術情報リポジトリ

Kyushu University Institutional Repository

\title{
Energy-Efficient Embedded System Design at 90nm and Below : A System-Level Perspective
}

Ishihara, Tohru

System LSI Research Center, Kyushu University

http://hdl. handle. net/2324/8987

出版情報: Lecture Notes in Computer Science. 4759, pp.452-465, 2008-01-19. Springer バージョン：

権利関係 : (c) 2008 Springer 


\title{
Energy-Efficient Embedded System Design at 90nm and Below - A System-Level Perspective -
}

\author{
Tohru ISHIHARA \\ System LSI Research Center, Kyushu University, \\ 3-8-33, Momochihama, Sawara-ku, Fukuoka, 814-0001, Japan \\ ishihara@slrc.kyushu-u.ac.jp \\ http://www.slrc.kyushu-u.ac.jp/
}

\begin{abstract}
Energy consumption is a fundamental barrier in taking full advantage of today and future semiconductor manufacturing technologies. This paper presents our recent research activities and results on estimating and reducing energy consumption in nanometer technology system LSIs. This includes techniques and tools for (i) estimating instantaneous energy consumption of embedded processors during an application execution, and (ii) reducing leakage energy in instruction cache memories by taking advantage of value-dependence of SRAM leakage due to within-die $V_{\text {th }}$ variation.
\end{abstract}

Key words: energy estimation, energy characterization, nanometer technology, leakage power, cache memory, compiler optimization, embedded system

\section{Introduction}

There is a wide consensus that the energy consumption is a fundamental barrier in taking full advantage of today and future semiconductor manufacturing technologies. As the demands of system integration, high performance, and low power operation have pushed chip vendors down to 90nm and below, NRE (nonrecurring engineering) costs and design complexity have increased significantly. A remedy for the NRE explosion is to reduce the number of developments and sell tens of millions of chips under a fixed hardware design. In such a situation, embedded software plays much more important role than today. Our research focus is mainly on software-oriented approaches to estimating and reducing the energy consumption of embedded real-time systems. In this paper, we present our recent research activities and results in the following two categories: estimating software energy consumption and reducing leakage energy of the memory subsystem. Firstly, we present a technique to estimate instantaneous energy consumption of embedded processors during an application execution. We train a per-processor energy-model which receives statistics from the processor instruction-set simulator (ISS) and gives the instantaneous energy consumption. Secondly, we show our 
technique for reducing leakage energy in instruction cache memories by taking advantage of value-dependence of SRAM leakage due to within-die $V_{t h}$ variation. We propose a technique to reduce the leakage power of ultra-leaky SRAM cells by storing less-leaky logic values to the corresponding SRAM cells in the cache.

The rest of this paper is organized as follows. The instantaneous energy estimation technique is presented in Section 2. Section 3 presents a techniques for reducing the leakage power of instruction cache memory by exploiting the value-dependence of SRAM leakage due to within-die $V_{t h}$ variation. Section 3 summarizes our approaches and concludes the paper.

\section{Software Power Estimation}

This section shows an overview of our energy characterization tool which helps designers in developing a fast and accurate energy model for a target processorbased system. We use a linear model for energy estimation and find the coefficients of the model using multiple linear regression analysis. For more detailed information of our tool see [1].

\subsection{Energy Characterization}

The energy consumption of a processor can be estimated using the following linear formula,

$$
E_{\text {estimate }}=\sum_{i=0}^{N} c_{i} \cdot P_{i}
$$

where $P_{i}$ 's, $c_{i}$ 's and $N$ are the parameters of the model, the corresponding coefficients and the number of parameters, respectively. The first step for the modeling is to find $P_{i}$ 's required for estimating the energy consumption of the target processor system. The $P_{i}$ 's should be parameters whose values can be easily obtained using a fast simulator like an instruction-set simulator (ISS). For example, $P_{i}$ 's can be the number of load and store instructions executed, the number of cache misses, etc. Once the required set of parameters is obtained, the next step is to find a training bench for the energy characterization. More detailed explanation for our method to generate the training bench is given in [1]. The final step is to find the coefficients, $c_{i}$ 's corresponding to the $P_{i}$ 's. This is done by using multiple linear regression analysis. The energy consumption $E_{\text {estimate }}$ is then calculated using Equation (1). Figure 1 shows an overview of our energy characterization flow. To obtain the reference energy values, we simulate the processor system at gate-level using the training bench. The training bench is divided into subsequences as shown in Fig.2. We refer to this sub-sequence as the instruction frame. The width is the same for all instruction frames. Since we perform gatelevel simulation and calculate the energy consumption values for all instruction frames, this step is time-consuming. However, it needs to be done only once for the characterization. In addition to this, the number of cycles simulated for the 


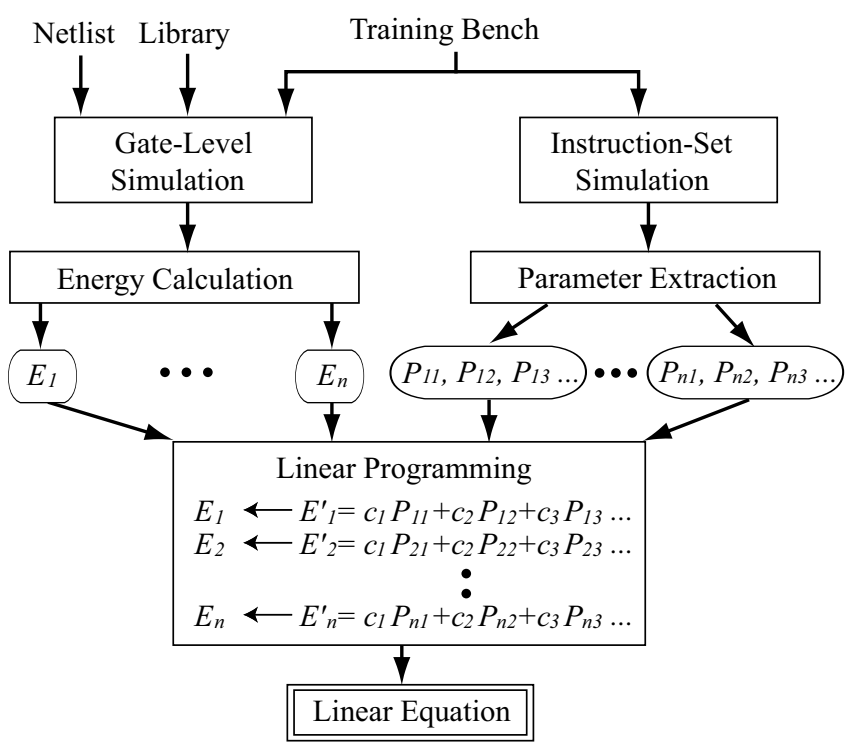

Fig. 1. Overview of Energy Characterization

training bench is much smaller than that for target application programs. More specifically, our training bench is simulated only about 500,000 cycles while the full simulation of the target application programs needs billions of cycles. Thus, total time required for the energy estimation using our approach is much smaller than that of gate-level or RT-level simulation-based approaches.

We, next, obtain an instruction trace for each application program using an instruction-set simulator. The traces are divided into small segments corresponding to instruction frames. $P_{i}$ 's should be parameters that can be easily extracted from instruction traces. For a set of $P_{i}$ 's, we find coefficients which minimize $\sum\left|E_{\text {estimate }}(i)-E_{\text {gate-level }}(i)\right|$, where $E_{\text {gate-level }}(i)$ and $E_{\text {estimate }}(i)$ are the energy consumption values obtained by gate-level simulation and Equa-

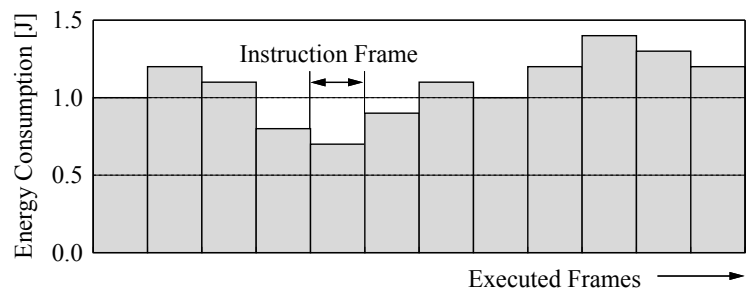

Fig. 2. An Example of Instruction Frame 
tion (1) for the $i^{\text {th }}$ instruction frame, respectively. Once the energy model is developed, the energy consumption of software running on the processor system can be estimated using a cycle-inaccurate instruction-set simulator (ISS) with the speed of 300,000 instructions per second.

\subsection{Experimental Results}

We target a system which consists of a CPU core, on-chip cache memories, and SDRAM as an off-chip main memory as shown in Fig.3. For the off-chip main memory, we assumed a Micron's SDRAM. We used an M32R-II processor and an SH3-DSP processor as CPU cores as follows.

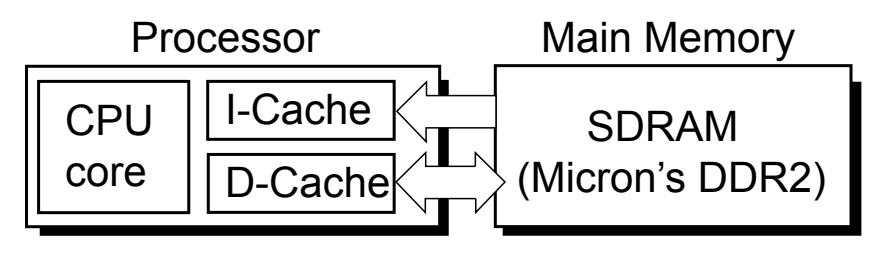

Fig. 3. A Target System Model

- M32R-II processor A 32-bit RISC microprocessor with 5-stage pipeline developed by Renesas Technology Corporation. It has 8KB 2-way set associative separate caches for instruction and data, a 32KB SRAM, and a 16-entry TLB on the chip.

- SH3-DSP processor A 32-bit RISC microprocessor developed by Renesas Technology Corporation. It has a digital signal processor core, a 32KB 4way set associative unified cache, a 128KB SRAM, and a 18KB SRAM on the chip.

We use 0.18 m CMOS standard cell library and SRAM module library for synthesizing the above two processors. Five benchmark programs shown in Table 1 are used in our experiment. Each benchmark program was simulated 1,000,000 instructions for evaluating our approach. Each instruction frame was 5,000 instructions long and there were total of 200 instruction frames.

First, we generate the Switching Activity Interchange Format (SAIF) file through gate-level simulation using $N C$-Verilog ${ }^{T M}$ from Cadence design systems. The SAIF file has the information about the values of the signals that change during simulation. Then, the energy consumption, $E_{i}$, is calculated for the $i^{\text {th }}$ instruction frame using DesignPower ${ }^{T M}$, a gate-level power calculation tool from SYNOPSYS. The average energy consumption per access for the instruction cache and the data cache are calculated using library data sheets. We used the Micron System Power Calculator [2] for calculating the energy consumption of SDRAM. Similarly, we generate an instruction trace using GNU C 
Table 1. Description of Benchmark Programs

\begin{tabular}{c||c}
\hline Benchmark Program & Program Description \\
\hline \hline JPEG & JPEG encoder version $6 \mathrm{~b}$ \\
\hline MPEG2 & MPEG2 encoder version 1.2 \\
\hline compress & File compression program \\
\hline FFT & Fast Fourier Transform \\
\hline DCT & Discrete Cosine Transform \\
\hline
\end{tabular}

debuggers for M32R-II and SH3-DSP processors. Note that the many of ISSs used in the GNU C debugger is cycle-inaccurate. We divide the instruction trace into small sub-traces each of which corresponding to an instruction frame and calculate the value of each parameter for each instruction frame. Finally, the optimal set of coefficients is found using $C P L E X^{T M}$, a Linear Programming solver form ILOG. The set of coefficients found minimizes the sum of estimation errors (i.e., $\left.\left|E_{i}-E_{i}^{\prime}\right|\right)$. After finding the optimal values of coefficients, we can use the linear equation to estimate the energy consumption for any instruction trace.

Table 2. CPU-Time for Characterization (Minutes)

\begin{tabular}{r||c|c}
\hline Target Processor & M32R-II & SH3-DSP \\
\hline \hline Gate-Level Simulation & 127 & 328 \\
\hline Power Calculation & 32 & 41 \\
\hline \hline LP Solver & $<1$ & $<1$ \\
\hline Total CPU Time & 160 & 370 \\
\hline
\end{tabular}

Table 2 shows the characterization results. The characterizations for M32R-II and SH3-DSP took 160 minutes and 370 minutes, respectively. Although this step is time-consuming, it needs to be done only once for a target processor system. We start with a set of predetermined parameters which include 82 parameters and select some of them for a given microprocessor. We generate the training bench so that the standard deviations of every predetermined parameter values are large enough and every correlation factors between any two parameters are small enough. The generated training benches are simulated 475,000 instructions and 140,000 instructions for M32R-II and SH3-DSP processors, respectively. If the value of the parameter multiplied by its corresponding coefficient is very small compared to the other values, the parameter will not be used due to its weak impact on the energy estimation. In addition to this, several parameters are merged into a single parameter if corresponding coefficient values are very close to each other. As a result, we chose 30 and 19 parameters for M32R-II and SH3-DSP processors, respectively. The parameters include the following: 
- The number of the following classes of instructions executed: 1) multiply, 2) divide, 3) multiply-add, 4) the other arithmetic operations, 5) logic, 6) shift, 7) register transfer, 8) load, and 9) store operations.

- The number of taken and untaken branches executed.

- The number of data and instruction cache misses.

- The number of times the instruction and data caches simultaneously miss.

- The number of times the read-after-write hazard occurs.

- The numbers of other events which cause a pipeline stall occur.

Table 3. Energy Estimation Results for M32R-II Processor

\begin{tabular}{|c||c|c|c|}
\hline & $\begin{array}{c}\text { Average } \\
\text { Error }\end{array}$ & $\begin{array}{c}\text { Maximum } \\
\text { Error }\end{array}$ & $\begin{array}{c}\text { Standard Deviation } \\
\text { of Error Percentage }\end{array}$ \\
\hline \hline JPEG & $2.70 \%$ & $10.32 \%$ & 2.76 \\
\hline JPEG_Opt & $0.69 \%$ & $16.46 \%$ & 6.17 \\
\hline MPEG2 & $1.54 \%$ & $3.97 \%$ & 0.94 \\
\hline MPEG2_Opt & $1.78 \%$ & $5.15 \%$ & 0.96 \\
\hline compress & $5.00 \%$ & $6.41 \%$ & 1.19 \\
\hline compress_Opt & $4.35 \%$ & $7.18 \%$ & 0.93 \\
\hline FFT & $1.55 \%$ & $6.87 \%$ & 0.92 \\
\hline FFT_Opt & $1.45 \%$ & $5.59 \%$ & 0.89 \\
\hline DCT & $1.42 \%$ & $8.58 \%$ & 0.72 \\
\hline DCT_Opt & $1.47 \%$ & $8.07 \%$ & 0.69 \\
\hline \hline Total & $2.74 \%$ & $16.46 \%$ & 2.82 \\
\hline
\end{tabular}

Table 4. Energy Estimation Results for SH3-DSP Processor

\begin{tabular}{|c||c|c|c|}
\hline & $\begin{array}{c}\text { Average } \\
\text { Error }\end{array}$ & $\begin{array}{c}\text { Maximum } \\
\text { Error }\end{array}$ & $\begin{array}{c}\text { Standard Deviation } \\
\text { of Error Percentage }\end{array}$ \\
\hline \hline JPEG & $3.17 \%$ & $11.89 \%$ & 3.11 \\
\hline JPEG_Opt & $6.33 \%$ & $10.02 \%$ & 2.79 \\
\hline MPEG2 & $1.32 \%$ & $3.41 \%$ & 0.98 \\
\hline MPEG2_Opt & $1.31 \%$ & $5.63 \%$ & 0.97 \\
\hline compress & $5.73 \%$ & $10.84 \%$ & 1.37 \\
\hline compress_Opt & $1.73 \%$ & $15.15 \%$ & 1.27 \\
\hline FFT & $1.27 \%$ & $3.25 \%$ & 0.76 \\
\hline FFT_Opt & $1.15 \%$ & $4.75 \%$ & 0.88 \\
\hline DCT & $1.12 \%$ & $2.20 \%$ & 0.46 \\
\hline DCT_Opt & $1.51 \%$ & $3.04 \%$ & 0.52 \\
\hline \hline Total & $2.47 \%$ & $15.15 \%$ & 2.45 \\
\hline
\end{tabular}

Average, maximum, and standard deviation of energy estimation errors for M32R-II and SH3-DSP processors are shown in Table 3 and 4, respectively. 
A suffix of each benchmark program "_Opt" represents that the program is compiled with a "-O3" option. The energy estimation error of our approach is on an average $2.7 \%$ and worst case $16.5 \%$ for M32R-II processor. For SH3-DSP processor, the error is on an average $2.5 \%$ and worst case $15.2 \%$. The accuracy of energy estimation is overall very good. The notable point is that the standard deviation of error percentage is very small. This shows that our estimation results have a similar trend to the gate-level results even though absolute errors are not very small in some cases.

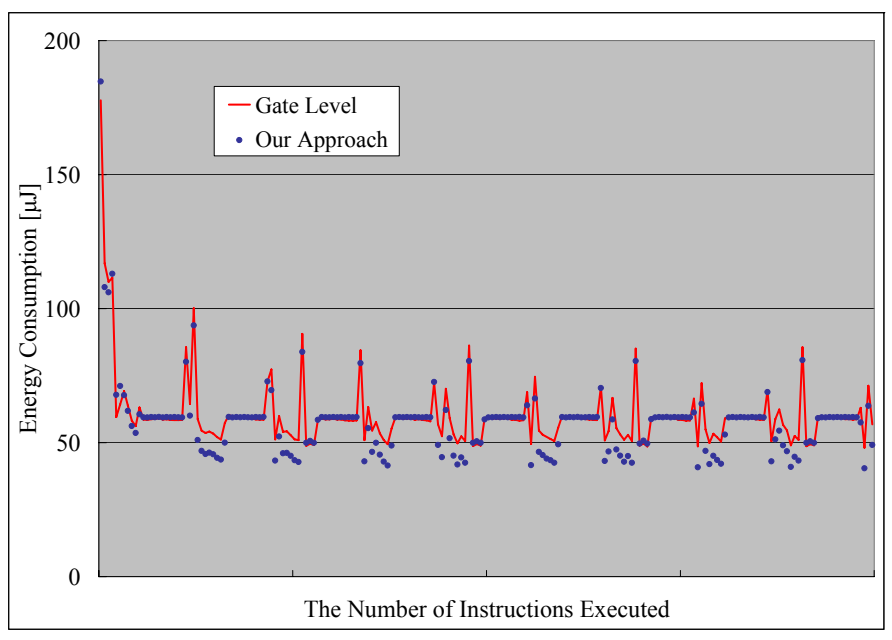

Fig. 4. Results for JPEG encoder run on M32R-II

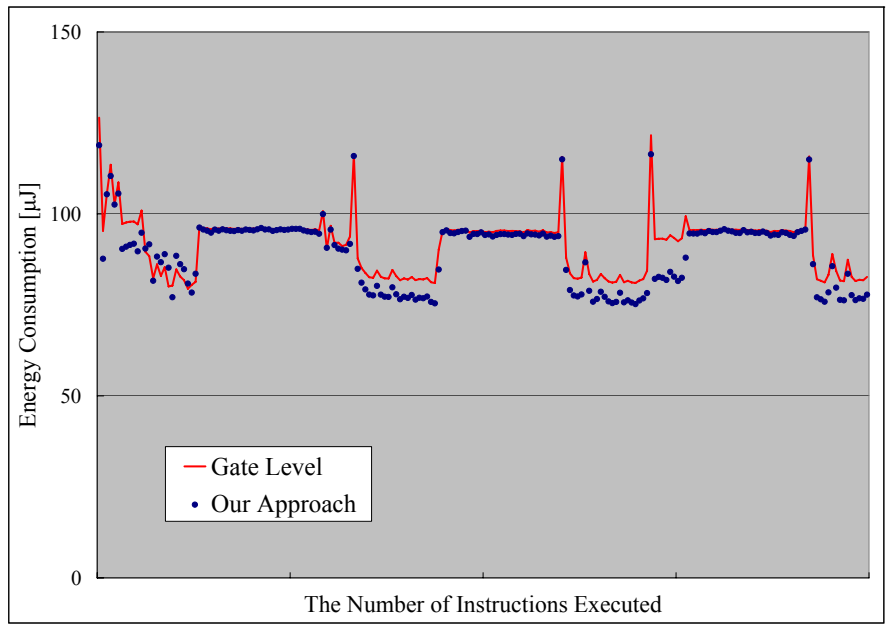

Fig. 5. Results for JPEG encoder run on SH3-DSP 
Figures 4 and 5 show the detailed results for JPEG encoder which runs on M32R-II and SH3-DSP processors, respectively. Horizontal and vertical axes represent instruction frame number and energy consumption per instruction frame, respectively. The energy consumption includes the energy for a CPU core, onchip caches, and off-chip SDRAM. As one can see, the estimation errors for every instruction frames are very small.

\subsection{Summary}

An energy characterization framework for processor-based embedded system is proposed. Experimental results obtained with two commercial microprocessors with their on-chip instruction and data caches, and an off-chip SDRAM demonstrated that the error of our technique is on an average $3 \%$ and worst case $16 \%$ compared to the gate-level estimation results. Our energy estimation method works very well even with a cycle-inaccurate simulator like a GNU debugger which is a de facto standard of software debugger. Once the model has been obtained, the energy consumption can be calculated with the speed of 300,000 instructions per second.

Today's SoC chips are usually implemented with off-the-shelf processor IPs. Even for those SoC chips, our method can accurately model the energy consumption since our tool does not need to know a detailed internal architecture nor the RTL description of the target processor. SoC vendors can easily generate an accurate energy model using our tool and provide it to their customers. This helps compilers or programmers to customize software codes to meet customers' needs for low power. Our future work will be devoted to extending the current framework to consider multi-core processor systems.

\section{Cache Leakage Reduction}

Random Dopant Fluctuation (RDF) [3] within the same die results in changes in the $V_{t h}$ of transistors. Transistors of cache SRAM cells are more affected since they have minimal physical channel area. The mismatch among $V_{t h}$ of transistors of a single SRAM cell results in different leakage currents depending on the value stored in the cell. Thus leakage of the cache memory can be reduced if the values with less leakage can be more often stored in each SRAM cell. We propose techniques to reduce instruction-cache leakage using this phenomenon.

\subsection{Value Dependence of SRAM Leakage}

When the SRAM cell is storing a 1 (Fig.6) only three transistors contribute to the total leakage (M1, M2, M5); when storing a 0, the other three transistors leak [4]. Since subthreshold leakage exponentially depends on $V_{t h}$, total leakage can be significantly different in the two states. Figure 7 shows probability distribution of SRAM leakage differences between two states. The standard deviation divided by the mean value of SRAM access latency is assumed to be a $5 \%$. The variation 


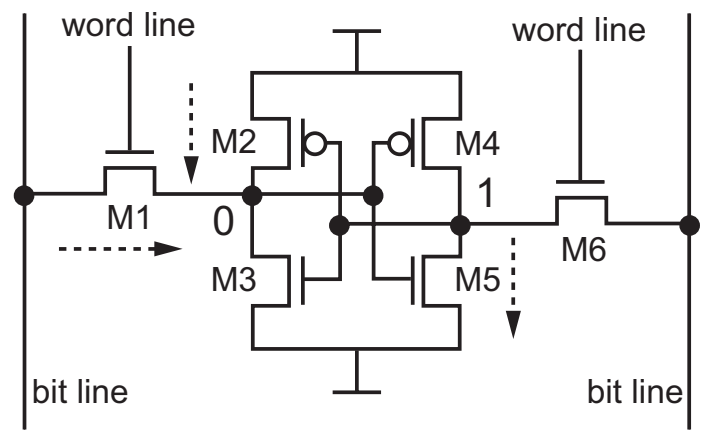

Fig. 6. Different transistors leak based on cell value

of SRAM access latency is closely corresponding to the $V_{t h}$ variation since the latency of an SRAM read access is almost linear to the $V_{t h}$ of an SRAM cell. Note that the values in Fig.7 are obtained from SPICE simulation for our original SRAM modules designed with a commercial $90 \mathrm{~nm}$ process technology. In our case, the total leakage power of a $4 \mathrm{~KB}$ SRAM module is $206 \mu \mathrm{W}$ at 75 degree Celsius if more-leaky values are stored to every SRAM cells. Contrary, if lessleaky values are stored to the every cells, the leakage power dissipated is only $53 \mu W$. If the logic values stored in the SRAM is random, the typical case leakage power is about $130 \mu \mathrm{W}$ which is an intermediate value between $206 \mu \mathrm{W}$ and $53 \mu W$. Therefore, we can save about $50 \%$ of the leakage power by storing lessleaky values to the SRAM cells. Another important observation is that only $10 \%$ of cells consume more than $50 \%$ of the total leakage power if there is $5 \%$ of delay variation (i.e., there is a $5 \% V_{t h}$ variation). In case of a $10 \%$ delay variation in the SRAM cells, more than $65 \%$ of the total leakage power is dissipated by $10 \%$ of the SRAM cells. This means that we can save a large amount of leakage power by storing less-leaky values to the small number of ultra-leaky SRAM cells.

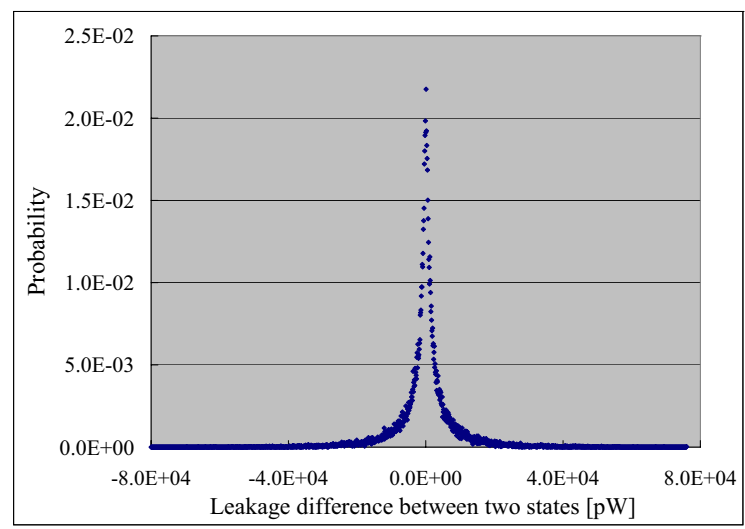

Fig. 7. SRAM leakage variation 


\subsection{Cache Leakage Power Reduction}

Our approach exploits an unused combination of existing flag bits (i.e., valid bit $=0$ and lock bit=1) to indicate ultra-leaky SRAM cells in a specific cacheline [5]. Suppose we have a 4 -way set associative cache with lock and valid bits as shown in Fig.8. If the lock bit of the way1 in the 5th cache-set is "1", the corresponding cache-line will not be used for replacement even in case of a cache miss. If the valid bit of way 1 in the 5 th cache-set is " 0 ", accessing to the corresponding block will always cause a cache miss. Thus, correct cache operation is guaranteed even in case that invalid values (i.e., less-leaky values) are locked in several cache lines. The basic idea of our approach is to reduce the leakage power of SRAM cells by locking less-leaky values in several cache lines which contain the ultra-leaky SRAM cells.

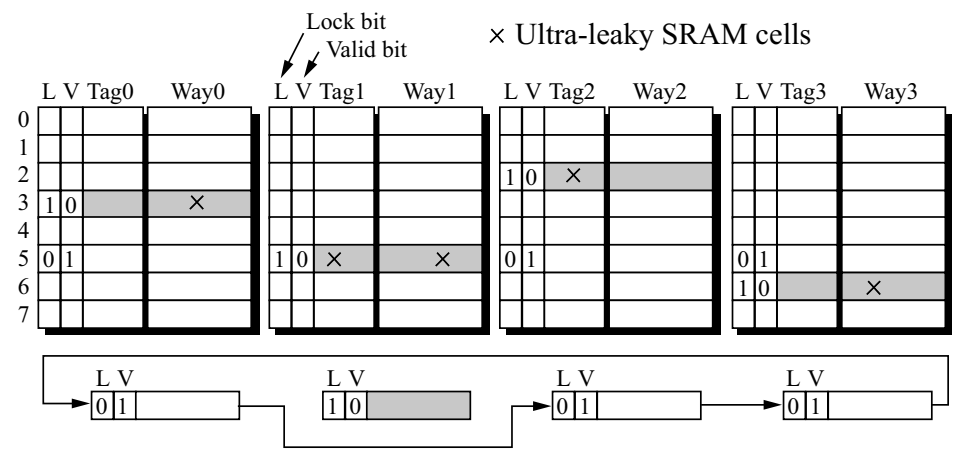

Cache Replacement Flow

Fig. 8. Cache locking function

Table 5 shows the results of leakage savings by our approach compared to the typical case where the values stored in the SRAM are random. The "powersaving cache-line" in Table 5 represents a cache line which stores less-leaky values in every bits in the cache line. The results show that we can save more than $20 \%$ of leakage power if the delay variation is more than $8 \%$ and if we store less-leaky values to the $25 \%$ of cache-lines.

Table 5. Leakage power reduction

\begin{tabular}{|l||c|c|c|c|}
\hline Standard deviation divided by mean latency & $4 \%$ & $8 \%$ & $12 \%$ & $16 \%$ \\
\hline \hline Percentage of power-saving cache-lines $=6.25 \%$ & $4.20 \%$ & $5.89 \%$ & $6.48 \%$ & $6.54 \%$ \\
\hline Percentage of power-saving cache-lines $=12.50 \%$ & $7.98 \%$ & $11.05 \%$ & $12.33 \%$ & $12.53 \%$ \\
\hline Percentage of power-saving cache-lines $=18.75 \%$ & $10.79 \%$ & $16.81 \%$ & $17.78 \%$ & $18.34 \%$ \\
\hline Percentage of power-saving cache-lines $=25.00 \%$ & $14.87 \%$ & $21.38 \%$ & $23.37 \%$ & $23.91 \%$ \\
\hline
\end{tabular}




\subsection{Cache Performance Improvement}

As shown in Table 2, the more cache lines are power-saving lines, the more leakage power can be saved. However, this leads to an increase of cache misses due to a decrease of an effective cache capacity. In this sub-section, we present a leakageaware code placement technique which reduces the performance degradation of a partially unused cache memory. Our approach is to modify the placement of basic blocks or functions in the address space so that the number of cache misses is minimized for a given cache having unused cache lines. More detail of our code placement algorithm is explained in [6]. An overview of our approach is depicted in Fig.9. First, we detect the locations of ultra-leaky SRAM cells in a cache memory. Next, our code placement technique generates the object code such that the number of cache misses can be lower than a given number even if several cache lines which contain the ultra-leaky cells are unused. Therefore, we perform recompilation only if the original object code does not satisfy the required performance for a specific chip. If the original object code or an object code previously generated for another chip can satisfy the required performance, we use it. Every time the chip is turned on, it executes an initialization step during which based on the information collected during test, ultra-leaky cache-lines are marked using lock and valid bits. Then the chip executes the compiled code.

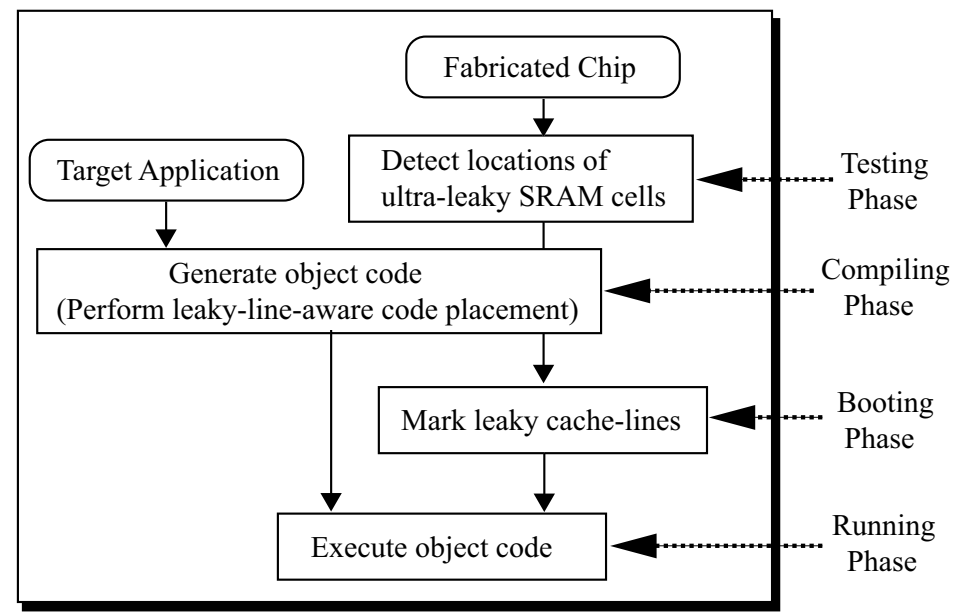

Fig. 9. Compiler optimization flow

\subsection{Experiments and Results}

We used three benchmark programs; compress version 4.0, JPEG encoder version 6b, and MPEG2 encoder version 1.2. All programs are compiled with "-O3" option. We used GNU C compiler and debugger for ARMv4T architecture to generate address traces. We used the following four types of cache memories: 
Cache-1 A 32Kb direct mapped cache with 128 cache-sets whose cache-line size is 32 bytes.

Cache-2 A $32 \mathrm{~Kb} 2$-way set-associative cache with 64 cache-sets whose cacheline size is 32 bytes.

Cache-3 A $32 \mathrm{~Kb}$ 4-way set-associative cache with 32 cache-sets whose cache line size is 32 bytes.

Cache-4 A $16 \mathrm{~Kb}$ 2-way set-associative cache with 32 cache-sets whose cache line size is 32 bytes.

In this experiment, we randomly chose ultra-leaky cache-lines (i.e., unused cache lines), and after that, we applied our leaky-line-aware code placement. We tried 480 different patterns of locations of ultra-leaky cache-lines for each benchmark program. Figures 10, 11, and 12 show the results for compress, JPEG encoder, and MPEG2 encoder, respectively. Black dots represent results of our leaky-line-aware code placement. In case of compress, there is no noticeable performance degradation even if $20 \%$ of total cache lines are unused. The results for the 4 -way set associative cache show that $25 \%$ of cache lines can be deactivated for all benchmark programs without noticeable performance degradation. In this case, we can save about $20 \%$ of leakage power consumption. This implies that it is better idea to deactivate several cache lines in a highly associative cache and to save leakage power of these deactivated cache lines by storing less-leaky values if there is a large process variation.
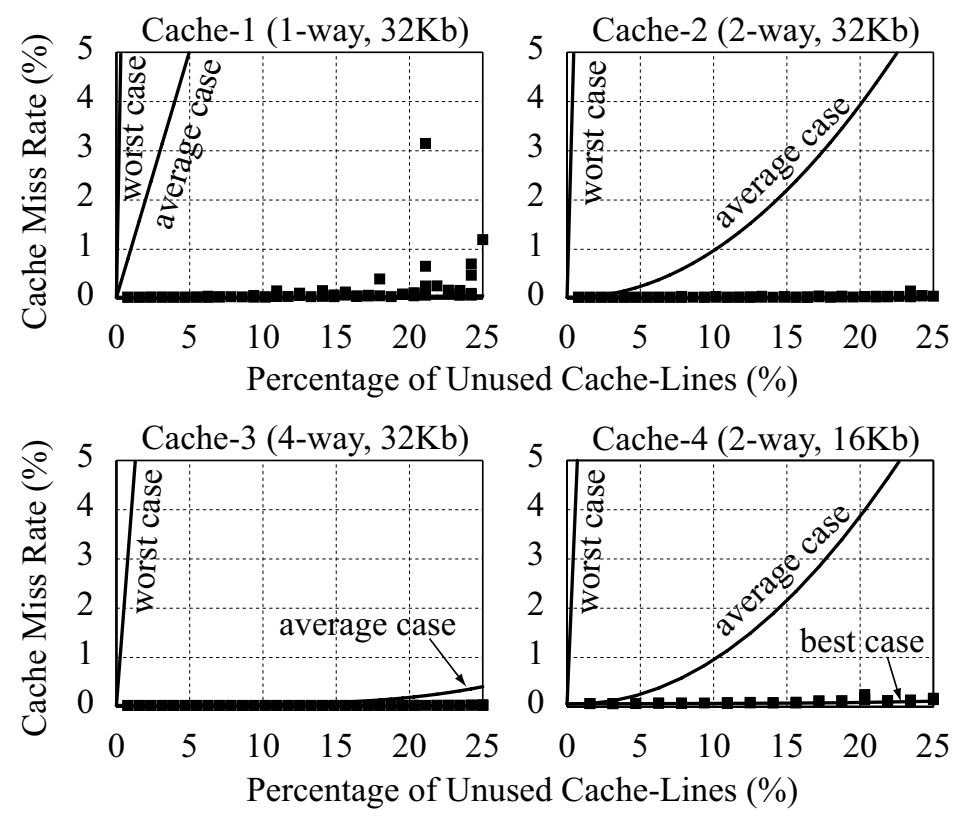

Fig. 10. Results for compress 

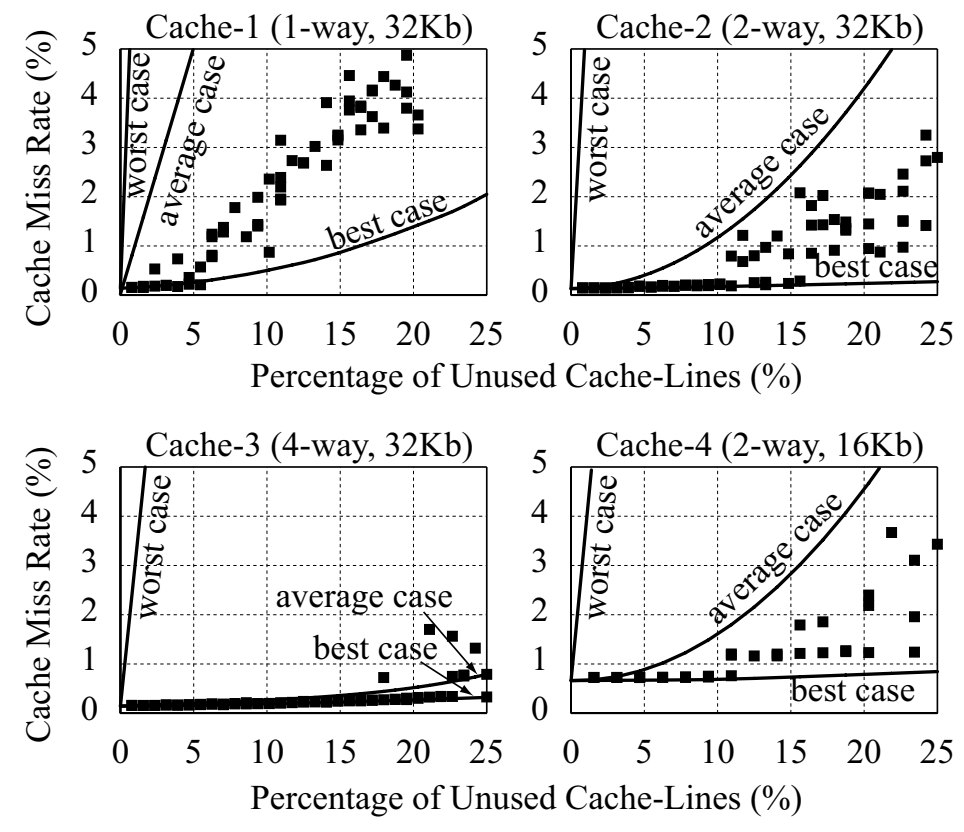

Fig. 11. Results for JPEG encoder
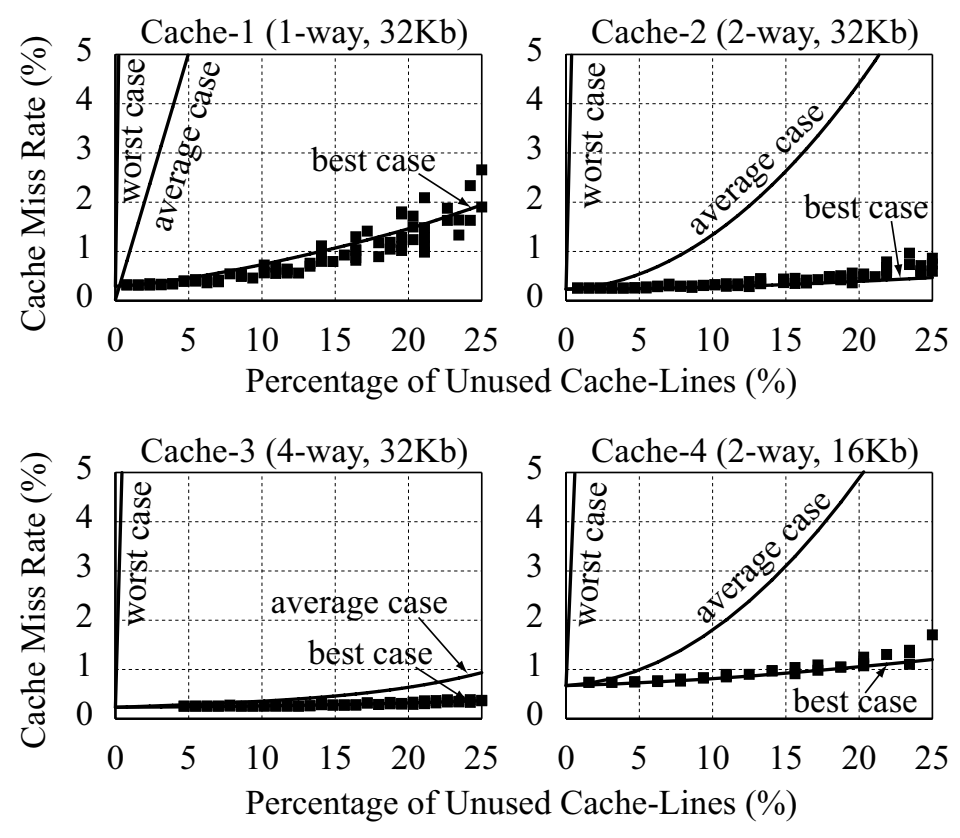

Fig. 12. Results for MPEG2 encoder 


\subsection{Summary}

In this section, a leaky-line-aware code placement technique is presented. Experiments demonstrate that our code placement technique offsets the impact of the reduced cache capacity on performance in most cases for 4-way set-associative caches even if $25 \%$ of cache lines are unused. In this case, about $20 \%$ of cache leakage power can be saved by locking less-leaky values in the unused lines.

\section{Conclusion}

As the transistor size scales down to $90 \mathrm{~nm}$ and below, non-recurring engineering (NRE) costs associated with mask set costs increase significantly. A remedy for the NRE explosion is to reduce the number of developments and sell tens of millions of chips under a fixed hardware design. In such a situation, embedded software plays much more important role than today. Our main focus is on software-oriented approaches to estimating and reducing the energy consumption of embedded real-time systems. We believe our research activities presented here will be a major trend in nanometer SoC design.

Acknowledgments This work is supported by VDEC, the university of Tokyo with the collaboration of Renesas Technology, STARC, Panasonic, NEC Electronics, Toshiba, ROHM, Toppan Printing, Cadence Design Systems, Synopsys and Mentor Graphics. This work is also supported by CREST program of JST.

\section{References}

1. D. Lee, T. Ishihara, M. Muroyama, H. Yasuura, and F. Fallah, "An Energy Characterization Framework for Software-Based Embedded Systems," in proc. of IEEE workshop on Embedded Systems for Real-Time Multimedia (ESTIMedia 2006), pp.59-64, Oct., 2006.

2. "The Micron System Power Calculator," http://www.micron.com/support/designsupport/tools/powercalc/powercalc.aspx

3. Y. Taur, and T.H. Ning, "Fundamentals of Modern VLSI Devices," in Cambridge University Press, 1998.

4. M. Goudarzi, T. Ishihara and H. Yasuura, "A Software Technique to Improve Yield of Processor Chips in Presence of Ultra-Leaky SRAM Cells Caused by Process Variation," in proc. of Asia and South Pacific Design Automation Conference (ASPDAC 2007), pp.878-883, Jan., 2007.

5. T. Ishihara and F. Fallah, "A Non-Uniform Cache Architecture for Low Power System Design," in proc. of International Symposium on Low Power Electronics and Design (ISLPED 2005), pp.363-368, Aug., 2005.

6. T. Ishihara and F. Fallah, "A Cache-Defect-Aware Code Placement Algorithm for Improving the Performance of Processors," in proc. of International Conference on Computer Aided Design (ICCAD 2005), pp.995-1001, Nov., 2005. 